\title{
ESTUDO DA INFLUENCIA DAS CONDIÇÕES OPERACIONAIS NA EFICIÊNCIA DO RECOBRIMENTO DE UREIA EM LEITO DE JORRO
}

\author{
G. F.WESTPHALEN ${ }^{1}$, F. DALLA NORA ${ }^{1}$, B. ZORZI ${ }^{1}$,E. ECHEVARRIA ${ }^{1}$ \\ T. P. da $\operatorname{COSTA}^{1}$ e G. S. ROSA ${ }^{1}$ \\ ${ }^{1}$ Universidade Federal do Pampa, Curso de Engenharia Química \\ E-mail para contato: juza.westphalen@hotmail.com
}

\begin{abstract}
RESUMO - A ureia é o fertilizante nitrogenado de maior demanda no país. Entre suas limitações tem-se as elevadas perdas do nitrogênio por volatilização, o que motiva estudos sobre alternativas para de amenizar essas perdas. Este trabalho teve como objetivo verificar a influência das condições operacionais na eficiência do processo de recobrimento da ureia em leito de jorro. Foi utilizado um planejamento fatorial em estrela com ponto central tendo como variáveis a temperatura do ar e a vazão de suspensão. Verificou-se que para um tempo fixo de processo de $20 \mathrm{~min}$, a faixa obtida para a eficiência do recobrimento foi de 17 a 46 $\%$.Observou-se que os efeitos linear e quadrático da vazão foram os mais pronunciados para a eficiência do processo, sendo que esta influência foi positiva, com um limite de confiança de $95 \%$.
\end{abstract}

\section{INTRODUÇÃO}

A ureia é caracterizada como um dos fertilizantes sólidos granulados de maior concentração de nitrogênio e é o fertilizante nitrogenado mais utilizado no Brasil, representando $75 \%$ do utilizado no país (Duarte, 2007). Entretanto, perdas por volatilização de até $78 \%$ do nitrogênio podem ocorrer após a aplicação de ureia sobre a superfície do solo sendo essas perdas dependentes muito em função do teor de matéria orgânica contida no solo (Rodrigues, 1986).

O uso de revestimentos pode reduzir a taxa de liberação de nitrogênio da ureia. Atualmente existem basicamente dois tipos de recobrimento, com enxofre ou com polímeros. No caso do recobrimento com polímeros, a liberação se dá através da difusão pela camada de cobertura, determinada pela característica química do polímero, da espessura, do processo de cobertura e da temperatura do meio. Os polímeros propiciam condições de controle e podem ser produzidos para sincronizar a liberação do nitrogênio de acordo com as necessidades nutricionais das plantas ao longo do ciclo de cultivo (Blaylock, 2007).

Entre os equipamentos que podem ser utilizados para realizar o recobrimento de partículas tem-se o leito de jorro. O sistema de leito de jorro consiste basicamente de uma coluna cilíndrica de base cônica, contendo partículas sólidas de um tamanho considerável, maiores que $1 \mathrm{~mm}$, e de um orifício de entrada do fluido localizado na parte inferior central da base cônica. Esta base cônica é utilizada para aumentar o movimento de sólidos e eliminar espaços mortos no fundo do leito. O vaso normalmente é um cilíndro circular, entretanto, o 
uso de um vaso completamente cônico foi prática comuns em alguns estudos (PASSOS et al., 1997).

O intuito desse trabalho foi verificar a influência das condições experimentais na eficiência do processo de recobrimento de ureia em leito de jorro, utilizando um planejamento fatorial em estrela.

\section{MATERIAIS E MÉTODOS}

Para o recobrimento da ureia foi utilizada uma suspensão aquosa de natureza polimérica capaz de fornecer uma superfície de recobrimento uniforme e de baixo valor comercial. Para isso, foram realizados testes com o objetivo de obter uma formação de filme liso e uniforme, e com adequada concentração de sólidos, visando obter espessura de filme suficiente, a fim de minimizar as perdas de nitrogênio por volatilização. A formulação da suspensão aquosa escolhida apresenta em peso: $0,5 \%$ de gelatina, $0,5 \%$ de amido, $2 \%$ do polímero comercial ColorSeed $^{\circledR}, 3 \%$ de plastificante (glicerol), $9 \%$ de talco e $85 \%$ de água.

Após foram realizados os ensaios de recobrimento com essa suspensão em leito de jorro. A Figura 1 apresenta o esquema da instalação experimental utilizada neste estudo.

Figura 1 - Representação da instalação experimental utilizada para os estudos de recobrimento.

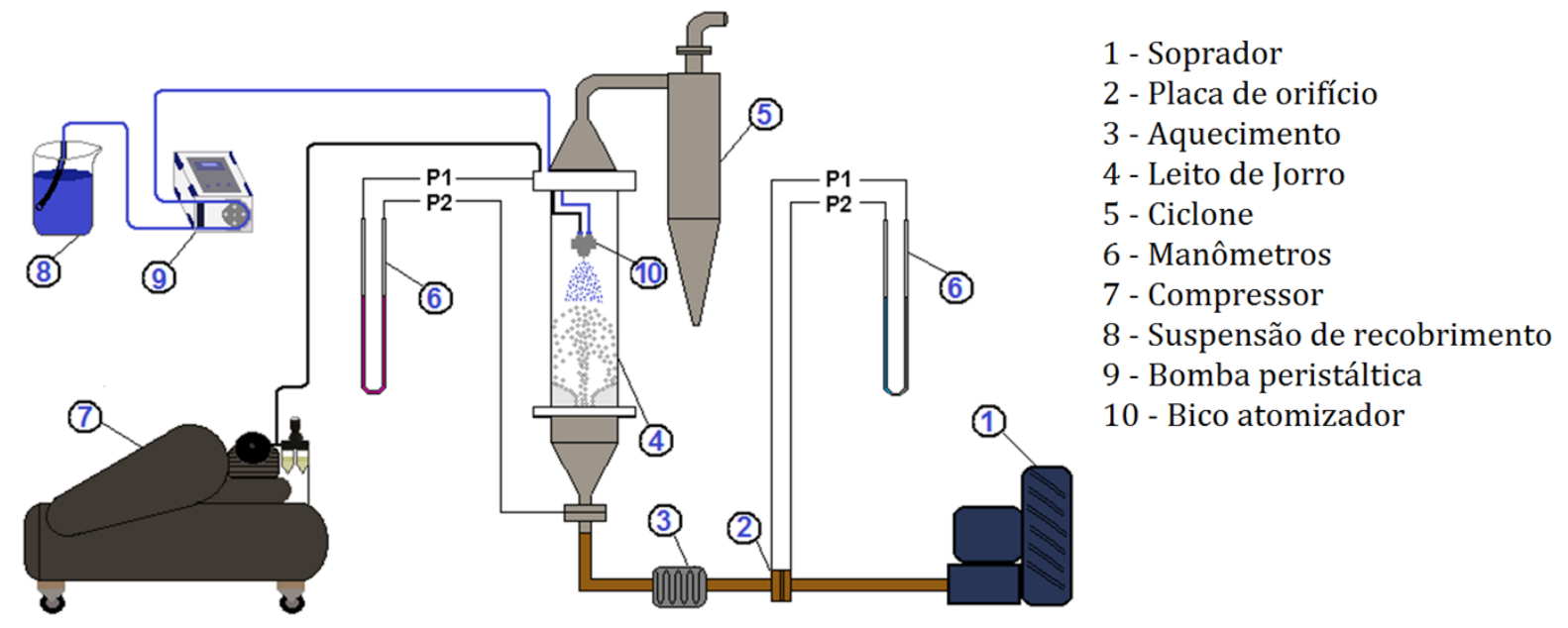

Através de ensaios fluidodinâmicos das partículas de ureia em leito de jorro foi definida a massa adequada para os ensaios de recobrimento. Os experimentos de recobrimento consistiram em abastecer o leito de jorro com a carga de ureia de 500 e em seguida foi iniciada a alimentação do ar de jorro através do soprador, sendo que este ar foi aquecido através de passagem em um conjunto de resistências. A vazão do ar foi ajustada no painel de controle e medida pela placa de orifício. Quando a temperatura de entrada do ar atingiu o valor desejado foi iniciada a alimentação da suspensão de recobrimento por meio de uma bomba peristáltica na vazão de atomização estabelecida.

A suspensão foi atomizada através de um bico atomizador de duplo fluido, localizado na parte superior do leito, onde vazão do ar de atomização era fornecida por um compressor com $0,5 \mathrm{kgf} / \mathrm{cm}^{2}$. O tempo de atomização da suspensão foi fixado em 20 min para todos os experimentos. 
Após a realização de testes preliminares de recobrimento foi elaborado um planejamento estrela com tréplica no ponto central a fim de analisar a influência das condições operacionais na eficiência do processo. As variáveis independentes foram: temperatura do ar de jorro $\left(\mathrm{T}_{\mathrm{ar}}\right)$ e vazão da suspensão de recobrimento $\left(\mathrm{W}_{\mathrm{s}}\right)$ e estão apresentadas na Tabela 1.

Tabela 1 - Variáveis e níveis utilizados.

\begin{tabular}{cccccc}
\hline Variáveis & \multicolumn{5}{c}{ Níveis } \\
\cline { 2 - 6 } independentes & $-1,41$ & -1 & 0 & 1 & 1,41 \\
\hline $\left.\mathrm{T}_{\mathrm{ar}}{ }^{\circ} \mathrm{C}\right)$ & 60,9 & 65 & 75 & 85 & 89,1 \\
$\mathrm{~W}_{\mathrm{s}}(\mathrm{mL} / \mathrm{min})$ & 7,8 & 9 & 12 & 15 & 16,2 \\
\hline
\end{tabular}

A eficiência do processo de recobrimento foi calculada a partir da Equação 1.

$\eta=\frac{M_{f}-M_{\circ}}{W_{s} \cdot C_{s} \cdot t \cdot \rho_{s}}$

Em que ${ }^{M_{f}}$ é a massa após o processo de recobrimento, $M_{0}$ é a massa inicial adicionada ao leito de jorro, ${ }^{W}$ é a vazão da suspensão de recobrimento, ${ }^{c}$ é a concentração de sólidos da suspensão, $\rho_{s}$ é a massa específica e $t$ é o tempo de processo.

A análise estatística dos resultados foi realizada com base em uma análise de variância (ANOVA) obtida utilizando o software Statistica7.

\section{RESULTADOS E DISCUSSÃO}

A Tabela 2 apresenta os resultados de eficiência obtidos para os ensaios realizados.

Tabela 2 - Eficiência do recobrimento.

\begin{tabular}{cccc}
\hline Ensaio & $\mathrm{T}_{\mathrm{ar}}$ & $\mathrm{W}_{\mathrm{s}}$ & Eficiência (\%) \\
\hline 1 & -1 & -1 & 17,26 \\
2 & -1 & +1 & 42,05 \\
3 & +1 & -1 & 33,84 \\
4 & +1 & +1 & 42,33 \\
5 & $-1,41$ & 0 & 31,42 \\
6 & $+1,41$ & 0 & 46,87 \\
7 & 0 & $-1,41$ & 38,01 \\
8 & 0 & $+1,41$ & 46,98 \\
$9(\mathrm{C})$ & 0 & 0 & 23,78 \\
$10(\mathrm{C})$ & 0 & 0 & 25,59 \\
$11(\mathrm{C})$ & 0 & 0 & 26,82 \\
\hline
\end{tabular}


Observando a Tabela 2 pode-se verificar que para um tempo fixo de processo de 20 min, a faixa obtida para o rendimento do recobrimento foi de 17 a $46 \%$. Também foi verificada uma boa reprodutibilidade do processo de recobrimento através dos resultados obtidos pelos experimentos 9 a 11, que correspondem aos pontos centrais. A eficiência do processo é considerada satisfatória industrialmente para valores acima de $65 \%$. Eficiências maiores poderiam ser alcançadas com tempos maiores de recobrimento, no entanto poderia aumentar a espessura do filme de recobrimento formado, prejudicando a liberação de nitrogênio.

Através da análise estatística dos resultados obtidos, verificou-se a influência das condições operacionais (variáveis independentes) na eficiência do processo de recobrimento. A Figura 2 mostra, para um limite de confiança de $95 \%(\mathrm{p} \leq 0,05)$, a influência dos efeitos principais e de suas interações através do diagrama de Pareto.

Figura 2 - Diagrama de Pareto.

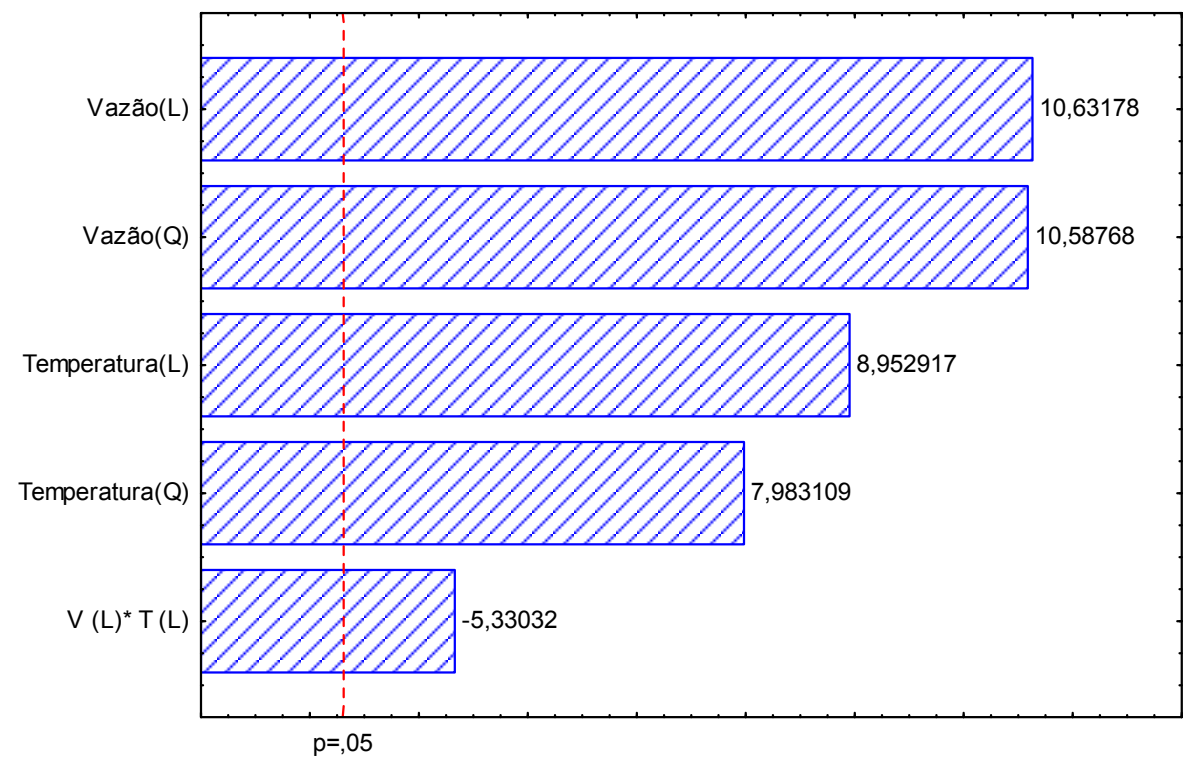

A partir dos resultados apresentados da Figura 2 observa-se que os efeitos linear e quadrático da vazão foram os mais pronunciados para a eficiência do processo, sendo esta influência positiva, ou seja, com um aumento da vazão de suspensão a eficiência do processo aumenta. Foi observada maior eficiência do processo para a maior vazão de suspensão, conforme o esperado, apesar da influência da temperatura.

Verificou-se também que o efeito da temperatura foi significativo. No caso da temperatura, constatou-se que o aumento desta também provoca uma maior eficiência do processo ocasionando a secagem do recobrimento sobre a partícula. Nas temperaturas mais baixas observou-se visualmente maior perda da suspensão na parede do leito.

Através da análise estatística dos resultados foi gerado um modelo polinomial de segunda ordem, apresentado na Equação 2 para a estimativa da eficiência do processo como uma função dos parâmetros estatisticamente significativos $(\mathrm{p}<0,05)$. Este modelo está na 
forma codificada, onde $\mathrm{T}$ e $\mathrm{W}$ correspondem, respectivamente, à temperatura e vazão da suspensão.

$$
\eta=25,398+4,848 \mathrm{~T}+5,134 \mathrm{~T}^{2}+5,745 \mathrm{~W}+6,809 \mathrm{~W}^{2}-4,073 \mathrm{TW}
$$

A significância do modelo estatístico foi verificada através da análise de variância (ANOVA), conforme apresentado na Tabela 3.

Tabela 3 - Análise de Variância.

\begin{tabular}{cccccc}
\hline & $\begin{array}{c}\text { Soma } \\
\text { Quadrática }\end{array}$ & $\begin{array}{c}\text { Graus de } \\
\text { liberdade }\end{array}$ & $\begin{array}{c}\text { Média } \\
\text { Quadrática }\end{array}$ & F calculado & F tabelado \\
\hline Regressão & 928,4309 & 5 & 928,4309 & 17,43 & 5,05 \\
Resíduos & 157,4424 & 5 & 53,2595 & & \\
Falta de ajuste & 152,7702 & 3 & 50,9234 & & \\
Erro puro & 4,6722 & 2 & 2,3361 & & \\
Total & 997,6231 & 10 & & & \\
\hline
\end{tabular}

Foi obtido um coeficiente de determinação (percentual de variação explicada) de93,06 \% para a regressão do modelo através da razão entre a soma quadrática da regressão pela soma quadrática total, em relação ao valor máximo explicável de 99,53\%. Isso significa que $93,06 \%$ da variação total em torno da média é explicada pela regressão, ficando $6,94 \%$ para os resíduos. Através do teste $\mathrm{F}$ observa-se que a regressão foi significativa ( $\left.F_{\text {calculado }}>F_{\text {tabelado}}\right)$. Este modelo foi usado para gerar a superfície de resposta para a eficiência do recobrimento, mostrada na Figura 3.

Figura 3 - Superfície de resposta e curvas de contorno para eficiência do processo.
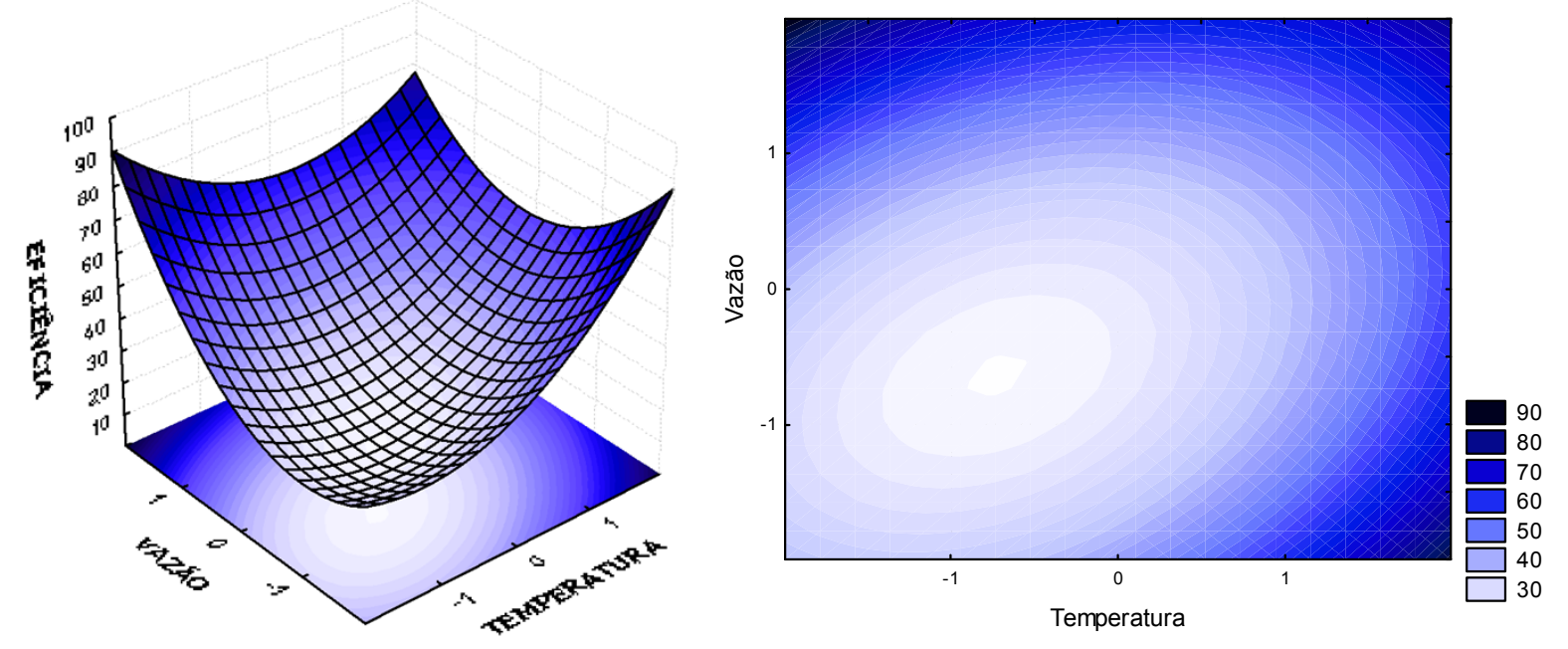

Observando a superfície e as curvas de contorno para a eficiência do processo pode-se verificar que a região que maximiza a eficiência do processo de recobrimento é próxima aos pontos extremos positivos, onde a temperatura e vazões são mais elevadas. 


\section{CONCLUSÃO}

Pode-se concluir que para um tempo fixo de processo de $20 \mathrm{~min}$, a faixa obtida para o rendimento do recobrimento foi de 17 a $46 \%$. Através da análise estatística dos resultados verificou-se que os efeitos da temperatura do ar, da vazão de suspensão e da interação de ambos foram significativos para a eficiência do processo.

Observou-se que os efeitos linear e quadrático da vazão foram os mais pronunciados para a eficiência do processo, sendo que esta influência foi positiva. Verificou-se também que o efeito da temperatura foi significativo, e que o aumento desta também provocou uma maior eficiência do processo, devido a secagem da suspensão do recobrimento sobre a partícula.

\section{NOMENCLATURA}

$C_{S}$
$M_{f}$
$M_{o}$
$t$
$W_{s}$
$\eta$
$\rho_{s}$

concentração de sólidos massa final de sólidos secos massa inicial de sólidos secos tempo vazão da suspensão eficiência massa específica da suspensão
$[-]$ $[\mathrm{mL} / \mathrm{min}]$

[\%]

$\left[\mathrm{g} / \mathrm{cm}^{3}\right]$

\section{REFERÊNCIAS BIBLIOGRÁFICAS}

BLAYLOCK, A. Novos Fertilizantes Nitrogenados: O Futuro dos Fertilizantes Nitrogenados de Liberação Controlada. Informações Agronômicas, Piracicaba, n. 120, p. 8-10, dez. 2007.

DUARTE, D. S. A. Perdas de amônia por volatilização em solo tratado com ureia, na presença de resíduos culturais. PPG-ESA LQ/USP - Dissertação de Mestrado Piracicaba - SP, 64 f. 2007.

GELDART, D. Types of gás fluidization, Powder Technology, v.7, p. 285-292, 1973.

MATHUR, K. B.; EPSTEIN, N. Spouted Beds.1.ed London: Academic Press, p.304, 1974.

PASSOS, M. L.; MASSARANI, G.; FREIRE, J. T.; MUJUMDAR, A. S. Drying of pastes in spouted beds of inert particles: design criteria and modeling. DryingTechnol., v.15, n.2, p. 605-624, 1997.

RODRIGUES, M. B. ; KIEHL, J. C. Volatilização de amônia após o emprego de ureia em diferentes doses e modos de aplicação. Revista Brasileira de Ciência do Solo, Viçosa, v.10, n.1, p. 37-43, 1986. 